amplitudes électriques $A, B, D$ (resp. C) se réfèrent à $M_{\text {trans }}=4 \mathrm{~kg}$ (resp. $1 \mathrm{~kg}$ ) adaptées sur $C_{a m p l i}=5 \mathrm{pF}$; l'adaptation mécanique, d'autre part, nécessite alors une masse totale de l'ordre de $100 \mathrm{~kg}$, avec laquelle le bruit thermique deviendrait équivalent à (1). Cependant, les signaux électriques étant nettement plus grands que le bruit d'un amplificateur convenable (FET), il devrait être possible, selon [2], d'arriver au moins à la limite (2), pourvu que le $Q_{\text {mécan }}$ ne soit pas trop faible.

Certaines combinaisons linéaires des signaux provenant des transducteurs individuels, indiquées également dans la figure 2, permettent l'étude séparée de l'excitation de chacune des harmoniques impaires de la vibration fondamentale. Ainsi, une antenne longue pourrait fonctionner comme analyseur de Fourier des signaux gravitationnels. Les performances d'un tel système dépendent essentiellement de la qualité des collages (adaptation mécanique avec un minimum de pertes d'énergie). Pour un test à échelle réduite, 2 barres de laiton ( $200 \mathrm{~mm}$ de long chacune, $\varnothing 9,0 \mathrm{~mm}$ ) furent collées à l'araldite conductrice sur les 2 côtés d'un transducteur à très faible $Q(\mathrm{PXE}-5 ; 20 \mathrm{~mm}, \varnothing 10,0 \mathrm{~mm}, Q \simeq 80)$. L'ensemble forme une ligne de transmission de $L=0,42 \mathrm{~m}$, dans laquelle une impulsion rapide peut être stockée durant plusieurs msec grâce à la réflexion parfaite aux extrémités. Le $Q$ du système monte de 450 pour l'oscillation fondamentale $\left(f_{1}=4,1 \mathrm{kHz}\right)$ jusqu'à un maximum de 1800 à $79 \mathrm{kHz}$ $\left(\approx 19 f_{1}\right)$. On en conclut qu'une antenne de $L=5 \mathrm{~m}$ couvrirait, avec un $Q$ suffisamment élevé, la bande spectrale de 500 à $4500 \mathrm{~Hz}$. Un article plus détaillé paraîtra ailleurs.

\title{
Bibliographie
}

[1] J. Weber, Evidence for Discovery of Gravitational Radiation, Phys. Rev. Lett. 22, 1320 (1969).

[2] G. W. Gibbons and S. W. Hawking, The Detection of Short Bursts of Gravitational Radiation (à paraître dans Phys. Rev.).

[3] D. MAEDER, Transmission-Line Analysis of Gravitational Pulse Detector Response Functions (à paraître dans Nucl. Instr. Meth.).

\section{Verstärkung und Superstrahlung von blitzlichtgepumpten Farbstofflasern}

von H. M. Gassmann und H. Weber

(Institut für angewandte Physik, Universität Bern)

Eine ausfürliehe Arbeit erscheint in Z. angew. Math. Phys. 22, Heft 5 (1971).

\section{Modellrechnungen von Sonnenausbrüchen mit kurzen Abklingzeiten}

von K. FLÜCKTGER

(Institut für angewandte Physik der Universität Bern)

Aus radioastronomischen Beobachtungen in den Jahren 1969 und 1970 bei einer Frequenz von $10,5 \mathrm{GHz}$ konnten impulsive Sonnenausbrüche mit Abklingzeiten von nur einigen Sekunden gefunden werden. Es wird versucht, diese kurzen Ausbrüche mit Hilfe von Modellrechnungen der Gyro-Synchrotronstrahlung von mittelrelativistischen und relativistischen Elektronen zu erklären. 